\title{
Allegories: decidability and graph homomorphisms*
}

\author{
Damien Pous \\ Univ Lyon, CNRS, ENS de Lyon, UCB Lyon 1, LIP \\ Lyon, France
}

\begin{abstract}
Allegories were introduced by Freyd and Scedrov; they form a fragment of Tarski's calculus of relations. We show that their equational theory is decidable by characterising it in terms of a specific class of graph homomorphisms.

We actually do so for an extension of allegories which we prove to be conservative: allegories with top. This makes it possible to exploit a correspondence between terms and $\mathrm{K}_{4}$-free graphs, for which isomorphisms were known to be finitely axiomatisable.
\end{abstract}

Keywords Allegories, Algebra, Graphs, Treewidth, Minors, Decidability, Homomorphisms

\section{Introduction}

In the nineties, Freyd and Scedrov proposed the notion of allegory [12], an axiomatisation of categories with some additional structure present in the category of relations (with sets as objects and binary relations as morphisms). Allegories arise in regular categories [18, Chapter A3]; they were also applied to circuit design [4] We show in this paper that their equational theory is decidable.

Forgetting the categorical structure, allegories form a finite and purely equational axiomatisation of the positive calculus of relations [3]. Their syntax is the following:

$$
u, v::=a|u \cdot v| u \cap v\left|u^{\circ}\right| 1
$$

Letter $a$ ranges over a set of variables. The first three operations intuitively denote relational composition $(\cdot)$, intersection $(\cap)$ and converse $\left({ }_{-}^{\circ}\right)$. The constant 1 corresponds to the identity relation.

We can associate to each term $u$ a labelled, directed graph $\mathrm{g}(u)$ with two designated vertices for input and output. In this construction, $a$ is a directed edge labelled with $a$, $\cdot$ is series composition of graphs, obtained by merging the output of the first graph and the input of the second one, $\cap$ is parallel composition, merging the inputs and the outputs of the two graphs respectively, $\left({ }_{-}^{\circ}\right)$ exchanges input and output, and 1 is the graph with no edge and a single vertex. For instance, the graphs of the terms $a \cdot\left(b \cap c^{\circ}\right) \cap d$ and $1 \cap a \cdot b$ are the following ones:
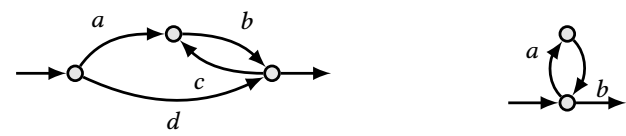

\footnotetext{
"This paper is the full version of [20] (Proc. LiCS'18), with appendices. This work has been supported by the European Research Council (ERC) under the European Union's Horizon 2020 programme (CoVeCe, grant agreement No 678157), and by the LABEX MILYON (ANR-10-LABX-0070) of Université de Lyon, within the program "Investissements d'Avenir" (ANR-11-IDEX-0007) operated by the French National Research Agency (ANR).

LICS '18, July 9-12, 2018, Oxford, United Kingdom

(C) 2018 Copyright held by the owner/author(s). Publication rights licensed to the Association for Computing Machinery.

This is the author's version of the work. It is posted here for your personal use. Not for redistribution. The definitive Version of Record was published in LICS '18: LICS '18: 33rd Annual ACM/IEEE Symposium on Logic in Computer Science, July 9-12, 2018, Oxford, United Kingdom, https://doi.org/10.1145/3209108.3209172.
}

\author{
Valeria Vignudelli \\ Univ Lyon, CNRS, ENS de Lyon, UCB Lyon 1, LIP \\ Lyon, France
}

A key result about allegories is that an inequation $u \geq v$ is universally valid for binary relations (i.e., it holds for all instantiation of its variables with binary relations) if and only if there is a graph homomorphism from $\mathrm{g}(u)$ to $\mathrm{g}(v)$. For instance, the inequation $a \cdot b \cap a \cdot c^{\circ} \geq a \cdot\left(b \cap c^{\circ}\right) \cap d$ can be proved by exhibiting the following homomorphism:

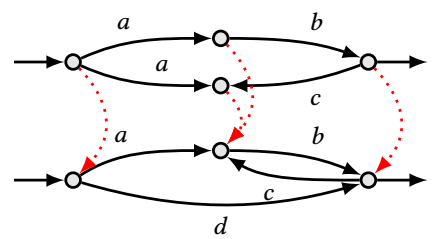

This characterisation was sketched by Freyd and Scedrov [12, page 208] and later proved by Andréka and Bredikhin [2, Theorem 1]. It actually appeared earlier under a different and more general form, to prove that the database problem of conjunctive queries containment is decidable [5, Lemma 13].

This characterisation however only applies to representable allegories, those allegories that are isomorphic to an algebra of concrete binary relations. Indeed, the equational theory of allegories is incomplete with respect to these models. Freyd and Scedrov give a counter-example [12, p. 210]: there are homomorphisms that correspond to inequations that are not derivable from the axioms of allegories. Incompleteness also follows from a general negative result by Andréka and Mikulás [3]: any finite first-order axiomatisation must be incomplete when the considered fragment contains at least the operations of composition, intersection and converse.

When looking at counter-examples to completeness, one can see that the problems always arise from homomorphisms that equate more than two vertices at a time. In fact, Freyd and Scedrov suggest that "the equations chosen as the definition of allegory happen to be precisely those that account for all containments obtainable by identifying the vertices two at a time" [12, p. 210].

We obtain decidability by proving this claim, which happens to be more difficult than expected. An attempt at exhibiting a proof was proposed in Gutierrez's doctoral dissertation [16]. However, his proof builds on a lemma which happens to be false and cannot be fixed [1,13]. Gutierrez also claimed decidability earlier, in a short abstract [15] where he proposes an alternative characterisation, but proofs are not available and some of his assertions seem highly nontrivial to prove. (See Appendix E). To the best of our knowledge, the problem is thus currently considered as open.

The key difficulty is that some graphs cannot be represented by a term. Thus, even though every homomorphism can be decomposed into a sequence of homomorphisms equating at most two vertices at a time, there is no guarantee that the intermediate graphs appearing in such a decomposition are the graphs of some terms. Consider for instance the graphs in Figure 1. There are homomorphisms from the outer graphs to the inner one, obtained by merging vertices depicted with the same symbol. While those three graphs are graphs of terms, we shall see that only the first one can be decomposed into 


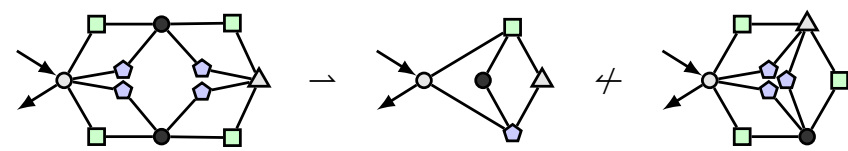

Figure 1. Valid and invalid homomorphisms. (The edges of the graph in the middle should be labelled with different letters and oriented arbitrarily; the orientation and labelling of the edges of the outer graphs are then determined by the two homomorphisms.)

a sequence where all intermediate graphs are graphs of terms (see Appendix A for more details), and thus corresponds to an inequality provable from allegories axioms.

We proceed in two steps.

1. First we solve the problem for allegories with top, that is, allegories extended with a neutral element for intersection, whose graph is the disconnect graph with no edges and two vertices, input and output. Doing so gives us more flexibility: there are more graphs that can be represented by a term (for instance, the disconnected ones), and there is a clear characterisation of the class of graphs of terms: they are precisely the graphs of treewidth at most two, or equivalently, the graphs excluding $\mathrm{K}_{4}$ as a minor. This move also makes it possible to exploit a recent axiomatisation of isomorphisms on such graphs [7]: we show that the corresponding axioms are derivable in allegories with top (Proposition 19), and we can then reason modulo isomorphisms. This latter possibility is crucial in most of our proofs.

2. Then we prove that allegories with top are a conservative extension of allegories: every equation over the signature of allegories that holds in all allegories with top actually holds in all allegories. We do so using model-theoretic means, by showing how to embed any given allegory into an allegory with top (Proposition 44). We solve in passing a problem that was left open in [7]: we give a finite axiomatisation of isomorphisms for connected $\mathrm{K}_{4}$-free graphs.

Outline and contributions We first recall the correspondence between terms and $\mathrm{K}_{4}$-free graphs [7] (Section 2) and setup tools to extract terms from graphs (Section 3). Then we define allegories with top and we prove laws that are required in the sequel (Section 4). Section 5 is devoted to our main contribution: there we characterise the inequational theory of allegories in terms of sequences of appropriate homomorphisms (Theorem 16). This characterisation leads to decidability (Section 6) and to a notion of normal form (Section 7). We finally proceed with the conservativity results (Section 8), which make it possible to lift our characterisation and decidability proof to pure allegories, and to provide a finite axiomatisation of isomorphisms for connected $\mathrm{K}_{4}$-free graphs.

The conservativity results and the equational proofs needed in the paper have been formally verified using the Coq proof assistant. The development can be downloaded and browsed online [21].

\section{Terms and graphs}

We let $a, b \ldots$ range over the letters of a fixed alphabet $A$. We consider labelled directed graphs with two designated vertices. We just call them graphs in the sequel. Note that we allow multiple edges between two vertices, as well as self-loops.

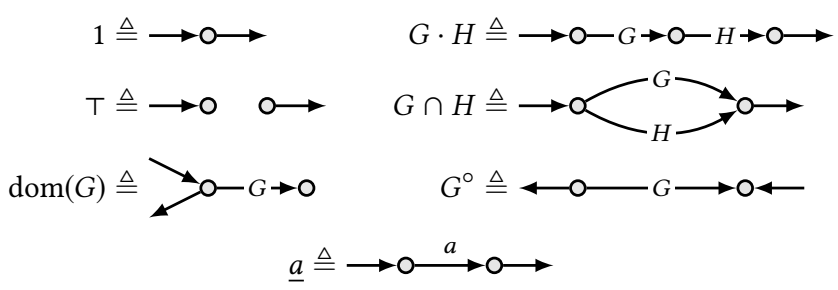

Figure 2. Graph operations.

Definition 1. A graph is a tuple $G=\langle V, E, s, t, l, l, o\rangle$, where $V$ is a finite set of vertices, $E$ is a finite set of edges, $s, t: E \rightarrow V$ are maps indicating the source and target of each edge, $l: E \rightarrow A$ is a map indicating the label of each edge, and $\iota, o \in V$ are the designated vertices, respectively called input and output.

Definition 2. A homomorphism from $G=\langle V, E, s, t, l, l, o\rangle$ to $G^{\prime}=$ $\left\langle V^{\prime}, E^{\prime}, s^{\prime}, t^{\prime}, l^{\prime}, \iota^{\prime}, o^{\prime}\right\rangle$ is a pair $h=\langle f, g\rangle$ of functions $f: V \rightarrow V^{\prime}$ and $g: E \rightarrow E^{\prime}$ that respect the various components: $s^{\prime} \circ g=f \circ s$, $t^{\prime} \circ g=f \circ t, l=l^{\prime} \circ g, \iota^{\prime}=f(\iota)$, and $o^{\prime}=f(o)$.

A surjective (resp. injective) homomorphism is a homomorphism whose two components are surjective (resp. injective) functions. A (graph) isomorphism is a surjective and injective homomorphism whose two components are bijective functions. We write $G \simeq G^{\prime}$ when there exists an isomorphism between graphs $G$ and $G^{\prime}$.

We consider the following signatures for terms and algebras:

$$
\Sigma=\left\{{ }_{2}, \cap_{2},{ }_{-1}^{\circ}, 1_{0}\right\} \quad \Sigma_{\mathrm{T}}=\Sigma \cup\left\{\mathrm{T}_{0}\right\} \quad \Sigma_{\text {dom }}=\Sigma \cup\left\{\operatorname{dom}_{1}\right\}
$$

We usually omit the symbol and we assign priorities so that the term $\left(a \cdot\left(b^{\circ}\right)\right) \cap c$ can be written just as $a b^{\circ} \cap c$.

Graphs form algebras for those signatures by considering the operations depicted in Figure 2, where inputs and outputs are represented by unlabelled ingoing and outgoing arrows. The operations composition $(\cdot)$ and intersection $(\cap)$ respectively correspond to series and parallel composition, converse $\left({ }_{-}^{\circ}\right)$ just exchanges input and output, and domain (dom(_)) relocates the output to the input.

By interpreting a letter $a \in A$ as the graph $a$ from Figure 2, one can thus associate a graph $\mathrm{g}(u)$ to every term over the considered signatures and with variables in $A$.

Observe that intersecting a graph with 1 amounts to merging its input and its output. As a consequence, the domain operation is derivable in the signature $\Sigma_{\top}$ thanks to the isomorphism below. Intuitively, relocating the output to the input can be implemented by first disconnecting the output (by multiplication with $T$ on the right), and then merging it with the input (by intersection with 1).

$$
\operatorname{dom}(G) \simeq 1 \cap G \top
$$

Accordingly, we will use the following shorthand when working with $\Sigma_{T}$-terms: $\operatorname{dom}(u) \triangleq 1 \cap u T$.

There are graphs which are not the graph of any term. For instance, this is the case for the following graphs, whatever the orientation and labelling of their edges.
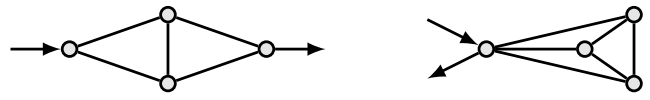

We now recall some standard graph theory notions, to state the characterisation of the graphs of $\Sigma_{T}$-terms from [7]. 
A simple graph is an unlabelled undirected graph with at most one edge between two vertices and without self-loops. We use standard notation and terminology from graph theory [9]. In particular, we denote by $k j$ a potential edge between two vertices $k$ and $j$; a $k j$-path is a (possibly trivial) path whose ends are $k$ and $j ; G+k j$ is the simple graph obtained from $G$ by adding the edge $k j$ if $k$ and $j$ were not already adjacent.

Definition 3. A minor of a simple graph $G$ is a simple graph obtained from $G$ by a sequence of the following operations: delete an edge or a vertex, contract an edge (i.e., delete it and merge its endpoints). A simple graph is $H$-free if $H$ is not one of its minors.

Robertson and Seymour's graph minor theorem [22], states that (simple) graphs are well-quasi-ordered by the minor relation. As a consequence, the classes of graphs of bounded treewidth [9], which are closed under taking minors, can be characterised by finite sets of excluded minors. Two simple and standard instances are the following ones: the graphs of treewidth at most one (the forests) are precisely those excluding the cycle with three vertices $\left(\mathrm{C}_{3}\right)$; those of treewidth at most two are those excluding the complete graph with four vertices $\left(K_{4}\right)[10]$.

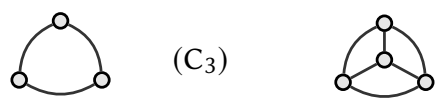

Definition 4. The skeleton of a graph $G$ is the simple graph $S$ obtained from $G$ by forgetting input, output, labelling, edge directions, edge multiplicities, and self-loops. The strong skeleton of $G$ is $S+\iota 0$ if $\iota \neq o$, and $S$ otherwise.

As an example, $\mathrm{K}_{4}$ is the strong skeleton of all instances of the graphs in (1).

Proposition 5 ([7, Corollary 26]). Let $G$ be a graph. The following are equivalent.

1. There exists a $\Sigma_{\top}$-term $u$ such that $G \simeq \mathrm{g}(u)$.

2. The strong skeleton of $G$ has treewidth at most two.

3. The strong skeleton of $G$ is $\mathrm{K}_{4}$-free.

In the sequel, we write $\mathrm{TW}_{2}$ for the set of graphs satisfying these conditions. The results from [7] also entail that Proposition 5 adapts to connected graphs just by restricting to $\Sigma_{\text {dom }}$-terms. ( $\sum$-terms alone are not enough, consider for instance the graph $\operatorname{dom}(\underline{a})$.)

\section{Parsing graphs}

Many different terms can denote the same graph. First because of associativity, commutativity, and neutral elements. But also, and more importantly, because of graphs whose input and output are equal. Consider for instance the graph in Figure 3, which is the graph of the five terms given on the left. Note that the terms in the second column do not exist in the syntax of pure allegories (the signature $\Sigma$ ): we need either the domain operation or its encoding through the constant $T$. The ability to write such terms in the syntax of allegories with top is crucial in Section 5.

We now prove a few results that allow us to extract terms from a given graph in $\mathrm{TW}_{2}$. We first focus on connected graphs.

Definition 6 (Primes, tests, petals, eyes). A graph $G$ is prime if it is connected and for all graphs $G_{1}, G_{2}, G \simeq G_{1} \cdot G_{2}$ entails $G_{1} \simeq 1$ or $G_{2} \simeq 1$. A graph is a test if its input is equal to its output. A petal is a prime test. An eye is a prime with distinct input and output.

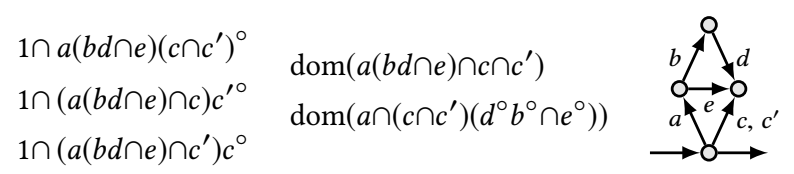

Figure 3. Different terms denoting the same connected graph.

The graphs of $1, a, a^{\circ}, a b \cap c, 1 \cap a$, and $1 \cap a b$ and $\operatorname{dom}(a)$ are all prime. The graphs of $a b, a(b \cap c), 1 \cap a \cap b c$ and $\operatorname{dom}\left((1 \cap a)\left(b \cap c^{\circ}\right)\right)$ are not, the latter two being the graph of $(1 \cap a)(1 \cap b c)$. A prime is either a petal or an eye. Petals can be characterised as follows.

Lemma 7. A test $G$ is a petal if either

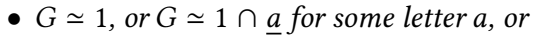

- $G$ has no self-loop on its input, is connected, and remains connected when removing the input.

As expected, every connected graph can be decomposed as a series composition of primes. This can typically be depicted as follows, where eyes are green and petals are yellow. The four depicted vertices are called checkpoints: they must be visited by any (undirected) path from the input to the output. Proper checkpoints are those different from input and output.

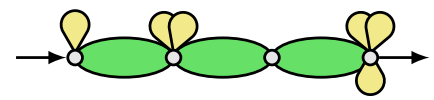

This decomposition is not unique however: there can be superfluous occurrences of 1 , and the order in which contiguous petals appear does not matter. If the starting graph belongs to $\mathrm{TW}_{2}$, then so do its prime components; this allows one to proceed recursively.

The following proposition makes it possible to decompose nontrivial eyes. This is a consequence of [7, Proposition 21(i)].

Proposition 8. Let $G \in \mathrm{TW}_{2}$ be an eye. Either $G$ consists of a single edge, or there are connected graphs $G_{1}, G_{2} \in \mathrm{TW}_{2}$ s.t. $G \simeq G_{1} \cap G_{2}$.

We write $G[k ; j]$ for the graph $G$ with input and output respectively set to $k$ and $j$. As illustrated in Figure 3, there can be several ways of extracting a term from a test. We shall mostly use the following observation, to resort to the case where input and output differ:

Observation 9. Let $G$ be a test and let $k$ be a vertex of $G$. We have $G \simeq \operatorname{dom}(G[\iota ; k])$.

To extract a term using this observation, one must however make sure that $G[l ; k]$ belongs to $\mathrm{TW}_{2}$. When $G$ is already known to be in $\mathrm{TW}_{2}$, one can use for $k$ any neighbour of the input: $G[l ; k]$ is necessarily in $\mathrm{TW}_{2}$ in such a case, since its strong skeleton is the same as that of $G$. This is how the two terms in the second column of Figure 3 are extracted. Other options are often possible, consider for instance the following graph:

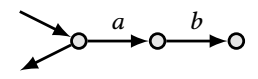

Choosing the neighbour of the input yields $\operatorname{dom}(a \operatorname{dom}(b))$, while choosing the other vertex yields $\operatorname{dom}(a b)$. Conversely, some options are forbidden: the topmost vertex in the graph of Figure 3 cannot be chosen, the strong skeleton of the resulting graph being $\mathrm{K}_{4}$.

Disconnected graphs can be parsed as follows:

Proposition 10. Let $G \in \mathrm{TW}_{2}$ be a disconnected graph. 


$$
\begin{aligned}
u \cap u & =u \\
u \cap(v \cap w) & =(u \cap v) \cap w \\
u \cap v & =v \cap u \\
u \cap \top & =u \\
u \cdot(v \cdot w) & =(u \cdot v) \cdot w \\
u \cdot 1 & =u \\
u^{\circ \circ} & =u \\
(u \cap v)^{\circ} & =u^{\circ} \cap v^{\circ} \\
(u \cdot v)^{\circ} & =v^{\circ} \cdot u^{\circ} \\
u v \cap u w & \geq u(v \cap w) \\
\left(v \cap w u^{\circ}\right) u & \geq v u \cap w
\end{aligned}
$$

Figure 4. Axioms of allegories with top $\left(A l l_{\top}\right)$.

1. If $G$ has a connected component $H$ which contains neither the input nor the output, then the graph $G^{\prime}$ obtained by removing $H$ from $G$ belongs to $\mathrm{TW}_{2}$ and for every vertex $k$ in $H$, we have $H[k ; k] \in \mathrm{TW}_{2}$ and $G \simeq G^{\prime} \cap \mathrm{T} H[k ; k] \mathrm{T}$.

2. Otherwise, $G$ has exactly two connected components $G^{\prime}$ and $G^{\prime \prime}$ respectively containing the input and the output, we have $G^{\prime}[l ; l], G^{\prime \prime}[o ; o] \in \mathrm{TW}_{2}$ and $G \simeq G^{\prime}[l ; \iota] \top G^{\prime \prime}[o ; o]$.

Proof. It suffices to show that the computed graphs are in $\mathrm{TW}_{2}$. This follows from the observation that their strong skeleton is always a subgraph of the strong skeleton of $G$, and the fact that the class of $\mathrm{K}_{4}$-free graphs is closed under taking subgraphs.

Remark 11. The occurrences of letters in a term are in one to one correspondence with the edges of its graph. As a consequence, if $C[]$ is a term context so that $C[a]$ is a term with a designated occurrence of the letter $a$, then given a term $u$, the graph of $C[u]$ is obtained from the graph of $C[a]$ by replacing the edge corresponding to the selected occurrence of $a$ with the graph of $u$.

\section{Allegories}

Definition 12. An allegory with top is a $\Sigma_{T}$-algebra satisfying the axioms in Figure 4, where an inequation of the form $u \geq v$ is a shorthand for the equation $u \cap v=v$. Given $\Sigma_{T}$-terms with variables in $A$, we write $\vdash_{A l l_{\top}} u=v$ when this equation is derivable from the axioms in Figure 4 (equivalently, when it holds in all allegories with top, for all interpretation of the variables). Similarly for inequations.

An allegory is a $\Sigma$-algebra satisfying those axioms but (A3). We define $r_{A l l} u=v$ accordingly.

Axioms (A0-A8) capture the most natural properties of the operators: intersection is idempotent, associative, commutative, and has $T$ as a neutral element (so that the derived relation $\geq$ is a partial order with $T$ as maximum element and intersection as meet); composition is associative and has neutral element 1 ; converse is an involution that reverses compositions. They entail $1^{\circ}=1, \mathrm{~T}^{\circ}=\mathrm{T}$, monotonicity of intersection and converse, and a notion of duality: every statement which holds universally also holds when reversing all compositions. We use such laws freely in the sequel.

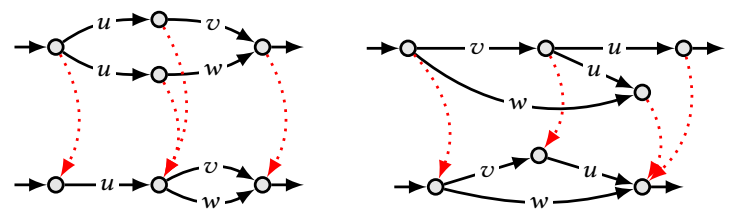

Figure 5. Surjective homomorphisms for Axioms (AD) and (AM).

Axiom ( $\mathrm{AD})$, semi-distributivity, is equivalent to monotonicity of composition on the right, and thus also on the left by duality, so that all operations are monotone in the end.

Axiom (AM) is called modular identity. This is the only unusual axiom. It generalises the notion of modularity for lattices [8]. Its dual is the following law:

$$
u\left(v \cap u^{\circ} w\right) \geq u v \cap w
$$

A symmetrical consequence of modular identity is the following inequation, known as Dedekind law:

$$
\left(v \cap w u^{\circ}\right)\left(u \cap v^{\circ} w\right) \geq v u \cap w
$$

The axioms of allegories are sound with respect to binary relations. As a consequence of the characterisation of representable allegories mentioned in the Introduction, we have:

Proposition 13. If $\vdash_{A l l_{\top}} u \geq v$ then there exists a homomorphism from $g(u)$ to $g(v)$.

Axioms (A1-A8) actually all correspond to graph isomorphisms. Idempotency $(\mathrm{A} 0)$ corresponds to an injective homomorphism from right to left, and to a surjective homomorphism from left to right; Axioms (AD) and (AM) correspond to the surjective homomorphisms depicted in Figure 5. Note that those three families of surjective homomorphisms may equate arbitrarily many vertices: the graph of $u$ might have many vertices in addition to input and output. (This issue will be addressed in Section 5.4.)

As a consequence of Proposition 13, we have $r_{A l l_{\top}} 1 \geq u$ if and only if $\mathrm{g}(u)$ is a test. We thus call tests the terms satisfying this condition. Equivalently, a test is a term that is provably equal to some term of the shape $1 \cap v$. Following notations from Kleene algebra with tests (KAT) [19], we let $\alpha, \beta$ range over tests.

Recall that $\operatorname{dom}(u) \triangleq 1 \cap u \top$ is a derived operation in allegories with top. Such an operation is not definable in pure allegories, but we can define a similar operation by setting $\operatorname{dom}^{\prime}(u) \triangleq 1 \cap u u^{\circ}$. Both domain operations are tests by definition, but the graphs of $\operatorname{dom}(u)$ and $\operatorname{dom}^{\prime}(u)$ are not isomorphic; they are depicted below.

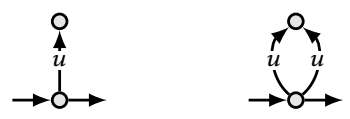

These operations are however interchangeable in allegories with top: we will prove the equation below after Proposition 14.

$$
\vdash_{A l l_{\top}} \operatorname{dom}(u)=\operatorname{dom}^{\prime}(u)
$$


Proposition 14. The following laws are derivable in allegories.

$$
\begin{gathered}
\operatorname{dom}^{\prime}(u \cap v)=1 \cap u v^{\circ} \\
\alpha(v \cap w)=\alpha v \cap w \\
\alpha \beta=\alpha \cap \beta=\beta \alpha \\
\alpha=\alpha^{\circ}=\alpha \alpha=\operatorname{dom}^{\prime}(\alpha) \\
\operatorname{dom}^{\prime}(u v)=\operatorname{dom}^{\prime}\left(u \operatorname{dom}^{\prime}(v)\right)
\end{gathered}
$$

Proof. We prove (3) by double inclusion. $1 \cap u v^{\circ} \geq \operatorname{dom}^{\prime}(u \cap v)$ follows by monotonicity from $u \geq u \cap v$ and $v^{\circ} \geq u^{\circ} \cap v^{\circ}$. For the other direction, we use Dedekind law (DD) with $w=1$ :

$$
1 \cap(u \cap v)\left(u^{\circ} \cap v^{\circ}\right) \geq 1 \cap\left(u v^{\circ} \cap 1\right)=1 \cap u v^{\circ}
$$

For Equation (4), we have

$$
\begin{aligned}
\alpha(v \cap w) & \geq \alpha\left(v \cap \alpha^{\circ} w\right) & \left(1 \geq \alpha^{\circ}\right) \\
& \geq \alpha v \cap w & \left(\text { by }\left(\mathrm{AM}^{\prime}\right)\right) \\
& \geq \alpha v \cap \alpha w & (1 \geq \alpha) \\
& \geq \alpha(v \cap w) & (\text { by (AD)) }
\end{aligned}
$$

It suffices to prove the first equation in (5), which follows from idempotency and (4): $\alpha \beta=\alpha(1 \cap \beta)=\alpha 1 \cap \beta=\alpha \cap \beta$.

For the first equation in (6), we have

$$
\begin{aligned}
(1 \cap u)^{\circ}=1 \cap 1 u^{\circ} & =\operatorname{dom}^{\prime}(1 \cap u) \\
& =\operatorname{dom}^{\prime}(u \cap 1)=1 \cap u 1^{\circ}=1 \cap u
\end{aligned}
$$

The other equations in (6) follow using (5). We get (7) as follows.

$$
\begin{aligned}
\operatorname{dom}^{\prime}\left(u \operatorname{dom}^{\prime}(v)\right) & \geq \operatorname{dom}^{\prime}\left(u \operatorname{dom}^{\prime}\left(u^{\circ} u v \cap v\right)\right) \\
& =\operatorname{dom}^{\prime}\left(u\left(1 \cap u^{\circ} u v v^{\circ}\right)\right) \\
& \geq \operatorname{dom}^{\prime}\left(u \cap u v v^{\circ}\right) \\
& =1 \cap u v v^{\circ} u^{\circ}=\operatorname{dom}^{\prime}(u v) \\
& \geq 1 \cap u\left(1 \cap v v^{\circ}\right) u^{\circ} \\
& =1 \cap u\left(1 \cap v v^{\circ}\right)\left(1 \cap v v^{\circ}\right)^{\circ} u^{\circ} \\
& =\operatorname{dom}^{\prime}\left(u \operatorname{dom}^{\prime}(v)\right)
\end{aligned}
$$

The above laws also hold in allegories with top. Equation (2) follows by instantiating $v$ with $T$ in (3).

\section{Graph theoretical characterisation}

We define a class of homomorphisms on $\mathrm{TW}_{2}$ graphs, that we will prove to characterise inequations in $A l l_{\top}$. Such homomorphisms, denoted by $\rightarrow$, are those that can be decomposed as a sequence of homomorphisms whose source and target are both in $\mathrm{TW}_{2}$ and equate at most two vertices. This is the case for the first homomorphism in Figure 1 (one must merge the black circles first, see Appendix A), but not for the second one: merging any two vertices with the same shape in the graph on the right yields a graph containing $\mathrm{K}_{4}$ as a minor.)

We let $R^{*}$ denote the reflexive-transitive closure of a relation $R$.

Definition 15. Define the following relations on graphs:

- $G \succ H$ if $G, H \in \mathrm{TW}_{2}$ and there is a surjective homomorphism $h: G \rightarrow H$ such that $h$ collapses at most two vertices;

- $G \hookrightarrow H$ if there is an injective homomorphism $h: G \rightarrow H$;

- $G \rightarrow H$ if $G(\succ \cup \hookrightarrow)^{*} H$;

- $G \rightleftharpoons H$ when $G \rightarrow H$ and $H \rightarrow G$.

$$
\begin{aligned}
1 \cap 1 & =1 \\
\operatorname{dom}(u \cap v) & =1 \cap u \cdot v^{\circ} \\
u \cdot \top & =\operatorname{dom}(u) \cdot \top \\
(1 \cap u) \cdot v & =(1 \cap u) \cdot \top \cap v
\end{aligned}
$$

Figure 6. Axioms for 2p-algebras (with (A1-A8)).

The class $\mathrm{TW}_{2}$ is closed under subgraphs, so $G \hookrightarrow H$ and $H \in \mathrm{TW}_{2}$ implies $G \in \mathrm{TW}_{2}$. Since we require $G$ and $H$ to be $\mathrm{K}_{4}$-free when $G \succ H$, we also have that $G \rightarrow H$ and $H \in \mathrm{TW}_{2}$ implies $G \in \mathrm{TW}_{2}$. (Note that when $h: G \rightarrow H$ is a surjective homomorphism equating exactly two vertices, then neither $G \in \mathrm{TW}_{2}$ implies $H \in \mathrm{TW}_{2}$ nor the converse. See Appendix B.)

Relation $\rightarrow$ is a preorder and $\rightleftharpoons$ is an equivalence relation. The remainder of this section is devoted to showing that the relation $\rightleftharpoons$ is sound and complete w.r.t. provability in $A l l_{\top}$ :

Theorem 16. We have $r_{A l l_{\top}} u=v$ if and only if $g(u) \rightleftharpoons g(v)$.

We start with the backward implication, for which it suffices to show that $g(u) \hookrightarrow g(v)$ entails $\vdash_{A l l_{\top}} u \geq v$ (Section 5.2) and $g(u) \succ g(v)$ entails $\vdash_{A l l_{\top}} u \geq v$ (Section 5.3). For both implications, a crucial preliminary step consists in dealing with isomorphisms (Section 5.1). Then we prove the forward implication (Section 5.4).

\subsection{Isomorphisms}

Definition 17 ([7, Section 3]). A $2 p$-algebra is a $\Sigma_{\top}$-algebra satisfying the axioms (A1)-(A8) from Figure 4 and the axioms (A9)-(A12) in Figure 6. We write $r_{2 p} u=v$ when two terms $u$ and $v$ are congruent modulo those axioms, or equivalently, when the equation holds in all $2 p$-algebras.

Note that idempotency $(\mathrm{A} 0)$ is not included in the axioms of $2 p$ algebra. $\mathrm{TW}_{2}$ is the free $2 p$-algebra; in particular, we have

Theorem 18 ([7, Corollary 34]). We have $r_{2 p} u=v$ iff $\mathrm{g}(u) \simeq \mathrm{g}(v)$.

We now observe that every allegory with top is a $2 p$-algebra.

Proposition 19. If $\vdash_{2 p} u=v$ then $\vdash_{A l l_{\top}} u=v$.

Proof. It suffices to prove axioms (A9)-(A12) from Figure 6. Axiom (A9) is a trivial instance of idempotency. Axiom (A10) follows from (3) and (2), and Axiom (A12) from (4). For Axiom (A11) we have $u \top \geq 1 \cap u \top$ and by (AM):

$$
u \top=1 \top \cap u \top \leq\left(1 \cap u \top \top^{\circ}\right) \top=\operatorname{dom}(u) \top
$$

Corollary 20. If $\mathrm{g}(u) \simeq \mathrm{g}(v)$ then $\vdash_{A l l_{\top}} u=v$.

This result is fundamental for the following proofs: it allows us to reason up to isomorphisms, and to freely choose the way we want to read a given graph. Recall for instance the five terms denoting the same graph in Figure 3; thanks to the above corollary, we know that those five terms are provably equal in allegories with top, so that we can freely replace one by the other.

\subsection{Injective homomorphisms}

Write $G \hookrightarrow_{v} H$ if there exists an injective homomorphism which is bijective on edges and adds exactly one vertex (i.e., such that there is exactly one vertex that is not in its range), and $G \hookrightarrow_{e} H$ if there 
exists an injective homomorphism which is bijective on vertices and adds exactly one edge (idem). We have

$$
\hookrightarrow=\hookrightarrow_{v}^{*} \hookrightarrow_{e}^{*}
$$

It thus suffices to show that $\hookrightarrow_{v}$ and $\hookrightarrow_{e}$ yield proofs in allegories with top. We exploit the result about isomorphisms to do so.

Proposition 21. If $\mathrm{g}(u) \hookrightarrow_{v} \mathrm{~g}(v)$ then $\vdash_{A l l_{\top}} u \geq v$.

Proof. Observe that $\mathrm{g}(u) \hookrightarrow_{v} \mathrm{~g}(v)$ entails $\mathrm{g}(u \cap \mathrm{T} \mathrm{T}) \simeq \mathrm{g}(v)$, and thus $\vdash_{A l l_{\top}} u \cap \mathrm{T \top}=v$ by Corollary 20 . We finally get

$$
A l l_{\top} \vdash u=u \cap \top \geq u \cap \top \top=v .
$$

Proposition 22. If $\mathrm{g}(u) \hookrightarrow_{e} \mathrm{~g}(v)$ then $\vdash_{A l l_{\top}} u \geq v$.

Proof. Suppose the added edge is labelled by $a$, and write $v=C[a]$ by selecting the corresponding occurrence of $a$ in $v$ (Remark 11). We have $g(u) \simeq g(C[\top])$, so $\vdash_{A l l_{\top}} u=C[\top]$ by Corollary 20 . We get $A l l_{\top} \vdash u=C[\top] \geq C[a]=v$ by monotonicity of all operations.

Note that in addition to Corollary 20, we are making a crucial use of the presence of $T$ in the syntax in the above two proofs. We could get rid of it when working with connected graphs, but this requires convoluted arguments (for instance, we can no longer handle vertices and edges separately and in an arbitrary order).

\subsection{Surjective homomorphisms}

Like above for injective homomorphisms, for two graphs $G, H \in$ $\mathrm{TW}_{2}$ write $G \succ_{v} H$ if there exists a surjective homomorphism which is bijective on edges and equates exactly two vertices, and $G \succ_{e} H$ if there exists a surjective homomorphism which is bijective on vertices and equates exactly two edges. We have

$$
\succ=\succ \underset{v}{=} \succ_{e}^{*}
$$

(Where $\succ_{v}^{=}$is the reflexive closure of $\succ_{v}$.) We now show that $\succ_{v}$ and $\succ_{e}$ yield proofs in allegories with top. This is easy for the latter, but the former relation requires a much deeper analysis.

Proposition 23. If $g(u) \succ_{e} \mathrm{~g}(v)$ then $\vdash_{A l l_{\top}} u \geq v$.

Proof. The only difference between $\mathrm{g}(u)$ and $\mathrm{g}(v)$ is that there are two parallel edges with the same label $a$ in $g(u)$ that are replaced by a single edge $a$ in $g(v)$. Let $v^{\prime}$ be the term obtained from $v$ by replacing by $a \cap a$ the occurrence of $a$ corresponding to this single edge in $g(v)$. We have $\vdash_{A l l_{\top}} v=v^{\prime}$ by idempotency. Now observe that $\mathrm{g}(u) \simeq \mathrm{g}\left(v^{\prime}\right)$, so that $\vdash_{A l l_{\top}} u=v^{\prime}$ by Corollary 20 .

Proposition 24. If $\mathrm{g}(u) \succ_{v} \mathrm{~g}(v)$ then $\vdash_{A l l_{\top}} u \geq v$.

Proof. Let $h$ be the surjective homomorphism from $g(u)$ to $g(v)$ collapsing exactly two vertices. We prove the statement by induction on $|g(u)|$, where $|G|$ is the lexicographic product of:

- the number of edges and vertices of $G$,

- 1 if $G$ is a test, and 0 otherwise.

We proceed by cases on the structure of $g(u)$.

1. $\mathrm{g}(u)$ is a disconnected graph. Then by Proposition 10 we have two cases:

a. $\mathrm{g}(u)$ has a connected component which contains neither the input nor the output. Hence, there are $w$ and $\alpha$ such that $\mathrm{g}(u) \simeq \mathrm{g}(w \cap \top \alpha \top)$. Let $k, k^{\prime}$ be the two collapsed vertices. If $k, k^{\prime}$ are either both in $\mathrm{g}(w)$ or both in $\mathrm{g}(\alpha)$, we derive the result by the inductive hypothesis. Suppose $k \in \mathrm{g}(w)$ and $k^{\prime} \in \mathrm{g}(\alpha)$. By isomorphism, we can assume that $k^{\prime}$ is the input of $\mathrm{g}(\alpha)$. Then $\mathrm{g}(u) \simeq \mathrm{g}(C[1 \cap \top \alpha \top])$, where 1 corresponds to vertex $k$, and $\mathrm{g}(v) \simeq \mathrm{g}(C[1 \cap \alpha])$. The result follows from $\vdash_{A l l_{\top}} \mathrm{T} \alpha \mathrm{\top} \geq \alpha$.

b. $\mathrm{g}(u)$ has exactly two connected components respectively containing the input and the output, and there are $\alpha$ and $\beta$ such that $\mathrm{g}(u) \simeq \mathrm{g}(\alpha \top \beta)$. Like in the previous case, we can conclude by induction if the collapsed vertices $k, k^{\prime}$ are either both in $\mathrm{g}(\alpha)$ or both in $\mathrm{g}(\beta)$. Suppose $k \in \mathrm{g}(\alpha)$ and $k^{\prime} \in \mathrm{g}(\beta)$. By hypothesis, collapsing $k$ and $k^{\prime}$ gives us a graph $\mathrm{g}(v)$ in $\mathrm{TW}_{2}$. Since vertex $k$ is connected to the input of $\mathrm{g}(\alpha)$ and $k^{\prime}$ is connected to the output of $\mathrm{g}(\beta)$, we derive that graphs $\mathrm{g}(\alpha)[l, k]$ and $\mathrm{g}(\beta)\left[k^{\prime}, o\right]$ are in $\mathrm{TW}_{2}$. Hence, there exist $w, x$ s.t. $\mathrm{g}(\alpha)[\iota, k] \simeq \mathrm{g}(w), \mathrm{g}(\beta)\left[k^{\prime}, o\right] \simeq \mathrm{g}(x)$. We have $\mathrm{g}(u) \simeq \mathrm{g}(w \top x)$ and $\mathrm{g}(v) \simeq \mathrm{g}(w x)$, and the result follows by $\vdash_{A l l_{\top}} w \top x \geq w x$.

2. $\mathrm{g}(u)$ is a connected test. Since $\mathrm{g}(u)$ has at least two vertices, there is some vertex $k$ adjacent to the input, but different from it. Then there is some term $w$ such that $\mathrm{g}(u) \simeq$ $\mathrm{g}(\operatorname{dom}(w))$ and $\mathrm{g}(u)[l ; k] \simeq \mathrm{g}(w)$. The existence of a homomorphism from $\mathrm{g}(u)$ to $\mathrm{g}(v)$ implies that $\mathrm{g}(v)$ is a test as well. Moreover, by the definition of homomorphism, $h(k)$ is either adjacent to the input in $g(v)$ or the input itself (in case the vertices identified by the homomorphism are exactly $k$ and the input of $\mathrm{g}(v)$ ). In both cases, there is a term $x$ such that $\mathrm{g}(v) \simeq \mathrm{g}(\operatorname{dom}(x))$ and $\mathrm{g}(v)[\imath ; h(k)] \simeq \mathrm{g}(x)$. The function $h$ is still a surjective homomorphism from $g(w)$ to $g(x)$ collapsing two vertices, since the only difference between $\mathrm{g}(\operatorname{dom}(w))$ and $g(w)$ is that the output has been relocated to $k$, and analogously the only difference between $g(\operatorname{dom}(x))$ and $g(x)$ is that the output has been relocated to $h(k)$. The graph $g(w)$ has the same number of vertices and edges as $\mathrm{g}(u)$, but has input different from output, so we can apply the inductive hypothesis to $g(w)$ and derive $\vdash_{A l l_{\top}} w \geq x$. Hence, $\vdash_{A l l_{\top}} \operatorname{dom}(w) \geq \operatorname{dom}(x)$.

3. $\mathrm{g}(u)$ is a connected graph with input different from output, and is not prime, i.e., there are $u_{1}, u_{2}$ both not equivalent to 1 such that $g(u)=g\left(u_{1}\right) \cdot g\left(u_{2}\right)$. Let $k, k^{\prime}$ be the vertices merged by the homomorphism. If either $k, k^{\prime}$ are both in $g\left(u_{1}\right)$ or they are both in $g\left(u_{2}\right)$ (possibly including the case when one of $k, k^{\prime}$ is the checkpoint between $g\left(u_{1}\right)$ and $g\left(u_{2}\right)$ in $g(u)$ ), we can apply the inductive hypothesis. Otherwise, $k$ is in $\mathrm{g}\left(u_{1}\right)$ and $k^{\prime}$ is in $\mathrm{g}\left(u_{2}\right)$, and neither of them is the checkpoint between $g\left(u_{1}\right)$ and $g\left(u_{2}\right)$. As discussed in Section 3, $\mathrm{g}(u)$ can be decomposed as a sequence of prime components $\mathrm{g}\left(u_{1}\right) \cdots \cdot \mathrm{g}\left(u_{n}\right)$, where each component is either a petal or an eye. W.l.o.g., we can assume that $k$ is in the first prime component, and that $k^{\prime}$ is in the last prime component (modulo the presence of petals equivalent to 1 ). If it were not the case, the inductive hypothesis could be applied as above. We consider different cases depending on whether $k, k^{\prime}$ are in a petal or in an eye.

a. Both $k$ and $k^{\prime}$ are in petals. Since $k$ and $k^{\prime}$ can be merged, and since $k^{\prime}$ is connected to the output, the output of the first petal can be relocated to $k$. Analogously, the input of the last petal can be relocated to $k^{\prime}$. Then there are $w, x, z$ s.t. $\mathrm{g}(u) \simeq \mathrm{g}(w \top x \cap z)$, as represented below, with $k$ the output of $\mathrm{g}(w)$ and $k^{\prime}$ the input of $\mathrm{g}(x)$, and $\mathrm{g}(v) \simeq$ $\mathrm{g}(w x \cap z)$. We conclude by $\vdash_{A l l_{\top}} \top \geq 1$. 


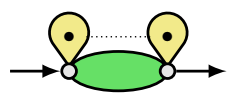

b. Both $k$ and $k^{\prime}$ are in an eye. Then (the strong skeleton of) $\mathrm{g}(u)$ has the following graph as a minor (the lower edge being the one added by the definition of strong skeleton).

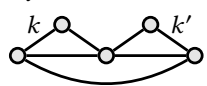

We thus get $\mathrm{K}_{4}$ by collapsing $k$ and $k^{\prime}$, which contradicts the fact that $\mathrm{g}(v)$ is the graph of a term.

c. $k$ is in a petal and $k^{\prime}$ is in a eye. We consider two cases:

i. Suppose the petal and the eye are contiguous, i.e., that there are no prime components between them (modulo the presence of 1). Then the petal is not equivalent to 1 , otherwise $k$ and $k^{\prime}$ would be in the same component. We have $\mathrm{g}(u) \simeq \mathrm{g}(\alpha w \cap x)$, with $k \in \mathrm{g}(\alpha)$ and $\mathrm{g}(w \cap x)$ the eye containing $k^{\prime}$, with $k^{\prime}$ strictly inside $\mathrm{g}(w)$ and $\mathrm{g}(x)$ containing at least one edge (this decomposition of an eye always exists by Proposition 8).

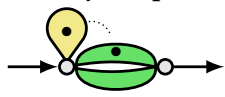

Then we have $\mathrm{g}(v) \simeq \mathrm{g}(z \cap x)$ with $\mathrm{g}(\alpha w) \succ_{v} \mathrm{~g}(z)$ and we conclude by the inductive hypothesis.

ii. Suppose the petal and the eye are not contiguous. If there are only petals (of which at least one not equivalent to 1) between them, we can move them by isomorphism before the petal containing $k$, and apply the inductive hypothesis. Hence, suppose there is at least one eye between the first and last prime component of $\mathrm{g}(u)$. We get different cases depending on where $k$ and $k^{\prime}$ are respectively located in the petal and in the eye.

A. If $k$ does not coincide with the input, then $g(u)$ has as a minor

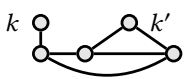

and by collapsing $k$ and $k^{\prime}$ we would have that $g(v)$ has $\mathrm{K}_{4}$ as a minor, which is a contradiction.

B. $k$ is the input and the eye containing $k^{\prime}$ has two parallel components $w, z$, for $k^{\prime} \in \mathrm{g}(w)$ and $\mathrm{g}(w)$ with at least one proper checkpoint. We have three cases:

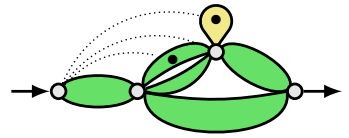

- $k^{\prime}$ is a proper checkpoint of $\mathrm{g}(w)$. Then $\mathrm{g}(u) \simeq$ $\mathrm{g}\left(x\left(w_{1} w_{2} \cap z\right)\right)$ with $k$ the input of $x$ and with $k^{\prime}$ the output of $w_{1}$, for $g(w) \simeq \mathrm{g}\left(w_{1} w_{2}\right)$. By collapsing $k, k^{\prime}$ we obtain $\mathrm{g}(v) \simeq \mathrm{g}\left(\left(x \cap w_{1}^{\circ}\right) z \cap w_{2}\right)$ and we derive in $A l l_{\top}$ :

$$
\begin{aligned}
x\left(w_{1} w_{2} \cap z\right) & \geq\left(x \cap w_{1}^{\circ}\right)\left(\left(x \cap w_{1}^{\circ}\right)^{\circ} w_{2} \cap z\right) \\
& \geq\left(x \cap w_{1}^{\circ}\right) z \cap w_{2}
\end{aligned}
$$

- $k^{\prime}$ is strictly in a petal of $g(w)$ with input a proper checkpoint of $\mathrm{g}(w)$. Then $\mathrm{g}(u)$ has as a minor

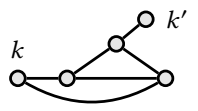

and by collapsing $k$ and $k^{\prime}$ we would have that $g(v)$ has $\mathrm{K}_{4}$ as a minor.

- $k^{\prime}$ is strictly in an eye of $g(w)$ having as output or input a proper checkpoint of $g(w)$. Then $g(u)$ has as a minor one of the following graphs:

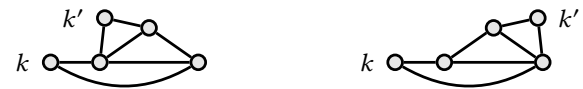

and, as in the previous case, by collapsing $k$ and $k^{\prime}$ we obtain $\mathrm{K}_{4}$.

4. $\mathrm{g}(u)$ is an eye not reduced to an edge. Then $\mathrm{g}(u) \simeq \mathrm{g}\left(u_{1}\right) \cap$ $\mathrm{g}\left(u_{2}\right)$ with both $\mathrm{g}\left(u_{1}\right)$ and $\mathrm{g}\left(u_{2}\right)$ containing at least one edge. If $k, k^{\prime}$ are in the same parallel component $\mathrm{g}\left(u_{i}\right)$, then we can apply the inductive hypothesis. Otherwise, suppose that they are respectively in $g\left(u_{1}\right)$ and $g\left(u_{2}\right)$. We can assume that both $g\left(u_{1}\right)$ and $g\left(u_{2}\right)$ have at least one proper checkpoint, since otherwise there would be a way to decompose $\mathrm{g}(u)$ into parallel components such that $k, k^{\prime}$ are in the same one. We have three cases:

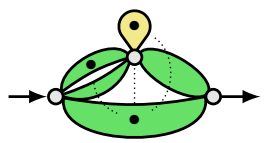

- $k, k^{\prime}$ are respectively proper checkpoints of $\mathrm{g}\left(u_{1}\right)$ and $\mathrm{g}\left(u_{2}\right)$. Then there are $w, w^{\prime}, x, x^{\prime}$ s.t. $\mathrm{g}(u) \simeq \mathrm{g}\left(w w^{\prime} \cap x x^{\prime}\right)$ and $g(v) \simeq \mathrm{g}\left((w \cap x)\left(w^{\prime} \cap x^{\prime}\right)\right)$, and we use Axiom (AD).

- $k$ is strictly in the petal of a proper checkpoint of $g(u)$ (or symmetrically for $\left.k^{\prime}\right)$. Then $\mathrm{g}(u)$ has as a minor

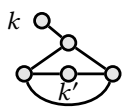

and by collapsing $k$ and $k^{\prime}$ we obtain $\mathrm{K}_{4}$.

- $k$ is strictly in an eye of $g\left(u_{1}\right)$ having as output or input a proper checkpoint of $g\left(u_{1}\right)$ (or symmetrically for $k^{\prime}$ ). Then $\mathrm{g}(u)$ has as a minor one of the following graphs
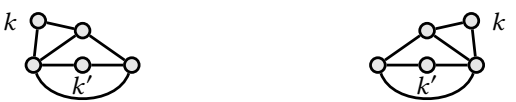

and, as above, by collapsing $k$ and $k^{\prime}$ we obtain $\mathrm{K}_{4}$.

5. It remains to consider the case when $\mathrm{g}(u)$ is an eye reduced to an edge. Then $\mathrm{g}(u) \simeq \mathrm{g}(a), \mathrm{g}(v) \simeq \mathrm{g}(a \cap 1)$ and the result follows by $\vdash_{A l l_{\top}} u \geq u \cap 1$.

Combining Corollary 20 and Propositions 21, 22, 23, and 24, we obtain that the relation $\rightarrow$ is sound for allegories with top:

Theorem 25. If $g(u) \rightarrow \mathrm{g}(v)$ then $\vdash_{A l l_{\top}} u \geq v$.

\subsection{Completeness}

For the converse of Theorem 25, we introduce an intermediate inequational presentation of allegories with top. This idea was already sketched in [12]; we give details here for the sake of completeness.

The inequational theory $A l l_{\mathrm{T}}^{\geq}$is generated by the axioms (A1)(A8) in Figure 4, where $u=v$ is a derived operator defined as $u \leq v$ and $v \leq u$, and the axioms in Figure 7. Atomic idempotency is an axiom scheme parameterised by letters $a \in A$. The point of this system is that its axioms all correspond to simple graph homomorphisms, that can easily be seen to belong to the relation $\rightarrow$ :

Lemma 26. For all axioms $u \geq v$ of $A l l \geq$, we have $g(u) \rightarrow g(v)$. 


$$
\begin{aligned}
u & \geq u \cap v & \text { (left inequality) } \\
u & \geq v \cap u & \text { (right inequality) } \\
1 \cap 1 & \geq 1 & (1 \text { idempotency) } \\
a \cap a & \geq a & \text { (atomic idempotency, for all } a \in A) \\
u \cdot v \cap x \cdot w & \geq(u \cap x) \cdot(v \cap w) & \text { (separated semi-distributivity) } \\
\left(u \cap w \cdot x^{\circ}\right) \cdot v & \geq u \cdot(v \cap x) \cap w & \text { (separated modularity) }
\end{aligned}
$$

Figure 7. Inequational presentation of allegories with top $\left(A l l_{\mathrm{T}}^{\geq}\right)$.

Proof. Axioms (A1)-(A8) from Figure 4 as well as idempotency for 1 correspond to graph isomorphisms. Inequality axioms correspond to injective homomorphisms, unless $v$ is a test and $u$ is not. In such a case, e.g., for left inequality, we have $g(u) \succ_{v} \mathrm{~g}(u) \cap 1 \hookrightarrow$ $\mathrm{g}(u \cap v)$. Atomic idempotency corresponds to $\succ_{e}$. Separated semidistributivity and separated modularity correspond either to $\succ_{v}$ or to $\simeq$. E.g., for separated semi-distributivity we have $\simeq$ if both $u$ and $x$ are tests or both $v$ and $w$ are tests, and we have $\succ_{v}$ otherwise.

Note that unrestricted idempotency, semi-distributivity and modular identity (Axioms (A0), (AD) and (AM) in Figure 4) could not be handled in such a way since they correspond to homomorphisms potentially equating many vertices and edges. That they are nevertheless captured by the relation $\rightarrow$ is obtained only a posteriori.

By further showing that $\rightarrow$ is 'closed under contexts', we deduce that the system $A l l \geq$ is sound for $\rightarrow$.

Lemma 27. For all term-contexts $C$, we have

$$
\text { 1. if } \mathrm{g}(u) \succ \mathrm{g}(v) \text { then } \mathrm{g}(C[u]) \succ \mathrm{g}(C[v]) \text {; }
$$

2. if $\mathrm{g}(u) \hookrightarrow \mathrm{g}(v)$ then $\mathrm{g}(C[u]) \hookrightarrow \mathrm{g}(C[v])$.

Proof. By induction on $C$. For the first item, when $C=[\cdot] \cap w$, if the merged vertices are the input and output of $u$ and if $w$ is a test then we have $\mathrm{g}(C[u]) \simeq \mathrm{g}(C[v])$, which is a special case of $\succ$.

Theorem 28. If $\vdash_{A l l} \geq u \geq v$ then $\mathrm{g}(u) \rightarrow \mathrm{g}(v)$.

It remains to show that the inequational presentation $A l l \geq$ is complete for allegories with top. We start by proving that (unrestricted) idempotency is derivable in $A l l_{\uparrow}^{\geq}$:

Lemma 29. For all terms $u$, we have $r_{A l l} \geq u \cap u=u$.

Proof. We prove $\vdash_{A l l} \geq \cap \cap u \geq u$ by induction on $u$. (The converse inequality trivially holds by the inequality axioms). The base cases are given by the axioms of $A l l_{\mp}^{\geq}$. For series composition we have $\vdash_{A l l \geq} u v \cap u v \geq(u \cap u)(v \cap v)$ by separated semi-distributivity, and then we conclude by the inductive hypothesis.

Theorem 30. If $\vdash_{A l l_{\top}} u \geq v$ then $\vdash_{A l l_{T}^{\geq}} u \geq v$

Proof. It suffices to derive modularity and semi-distributivity from their separated versions; this follows from Lemma 29.

Combining Theorems 25, 28, and 30 we finally obtain Theorem 16 .

\section{Decidability}

We now show that the relation $\rightarrow$ is decidable, and that there is a notion of normal form for allegories with top. The key observation is that surjective homomorphisms can always be applied first.

Lemma 31. We have the following inclusion: $\hookrightarrow \succ \subseteq \succ \hookrightarrow$.

Proof. Let $i: G \hookrightarrow G^{\prime}$ and $h: G^{\prime} \succ H$. The graph $h i(G)$ is $\mathrm{K}_{4}$-free, being a subgraph of $H$, and the function $g: G \rightarrow h i(G)$, defined as $h i$, is a surjective homomorphism collapsing at most two vertices. Indeed, function $g$ is trivially surjective, and either the vertices collapsed by $h$ are both in $i(G)$, in which case $g$ collapses them, or at least one of the collapsed vertices is not in $i(G)$, which implies that $g$ is an isomorphism. Therefore, we have $G \succ h i(G)$. Since $h i(G)$ injects in $H$, we conclude that $G \succ \hookrightarrow H$.

As a consequence, we obtain the following characterisation, which gives decidability:

Proposition 32. We have $G \rightarrow H$ iff $G \succ^{*} \hookrightarrow H$.

Corollary 33. The relation $\rightarrow$ is decidable.

Proof. It suffices to decide whether $G \succ^{*} \hookrightarrow H$. We have that the sets $\left\{G^{\prime} \mid G \succ^{*} G^{\prime}\right\}$ and $\left\{H^{\prime} \mid H^{\prime} \hookrightarrow H\right\}$ are finite and computable (up to isomorphism); it suffices to test whether they intersect.

One can actually get a non-deterministic polynomial algorithm: guess a sequence $G_{0}, \ldots, G_{n}$ of graphs obtained from $G=G_{0}$ by merging two vertices at a time, check that these graphs belong to $\mathrm{TW}_{2}$, compute the graph $H^{\prime}$ obtained from $G_{n}$ by merging all parallel edges with the same label (so that $G_{n} \succ_{e}^{*} H^{\prime}$ ) and check that $H^{\prime} \hookrightarrow H$. The latter test can be done in polynomial time once $G_{n}$ and thus $H^{\prime}$ are known to have bounded treewidth $[6,11,14]$.

Corollary 34. The equational theory of $A l_{\top}$ is in NP.

\section{Normal forms}

When studying homomorphism equivalence on graphs, one often uses the notion of core, those graphs where every endomorphism is an isomorphism. Every graph has a core, which is a minimal graph in its equivalence class modulo homomorphism equivalence [17]. One defines a similar notion here for allegories, using our restricted form of homomorphism equivalence $(\rightleftharpoons)$.

The normal form of a graph $G$, written $\operatorname{nf}(G)$ is a graph which is minimal w.r.t. the number of vertices and edges in its equivalence class modulo $\rightleftharpoons$. Normal forms are unique up to isomorphism:

Proposition 35. We have $G \rightleftharpoons H$ iff $\operatorname{nf}(G) \simeq \operatorname{nf}(H)$.

Define the following (computable) relation:

$$
G \leadsto H \triangleq G \succ^{*} H \hookrightarrow G
$$

Proposition 36. The relation $\leadsto$ is a confluent and terminating preorder and for all graphs $G$ we have $G \sim \operatorname{nf}(G)$.

Proof. That $\leadsto$ is a preorder follows from $\succ^{*}$ and $\hookrightarrow$ being preorders. Termination follows from the observation that the size of a graph decreases along $\succ$ (one must of course forbid trivial steps). Confluence is proved by induction on the sum of the sizes of the considered graphs, using Proposition 32 (see Appendix C). 
Gutierrez proves a similar result in the context of finite categories with an epi-mono factorisation system [16, Chapter 4.2]. One cannot reuse his result directly: the injective (resp. surjective) homomorphisms we use here are not exactly the mono (resp. epi) morphisms of the natural category associated to the relation $\rightarrow$.

To illustrate this rewriting system, consider the four graphs depicted below. We highlight pairs of vertices that can potentially be merged by representing them with the same symbol, using the same convention as in Figure 1 for labels and orientation of edges. $G_{4}$ is the normal form of the four graphs, and there are three ways of reaching it from $G_{1}$ : directly, or by going through $G_{2}$ or $G_{3}$.

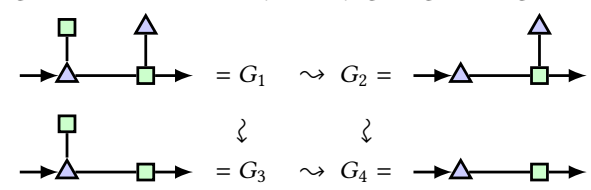

In this example, every attempt to collapse two vertices results in a graph in $\mathrm{TW}_{2}$. This is not always the case, as shown below.
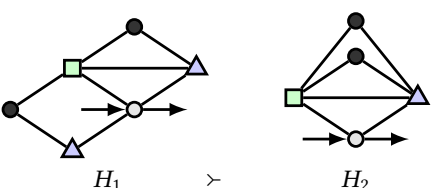

$\mathrm{H}_{2}$

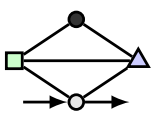

$\mathrm{H}_{3}$
$\mathrm{H}_{3}$ is the normal form of those three graphs. The unique homomorphism from $H_{1}$ to $H_{3}$ can be factorised through the graph $H_{2} \in \mathrm{TW}_{2}$ obtained by first merging the vertices depicted with triangles. Since $H_{3} \hookrightarrow H_{1}$, we deduce $H_{1} \leadsto H_{3}$. We also have $H_{2} \leadsto H_{3}$, but not $H_{1} \leadsto H_{2}$, since $H_{2}$ does not embed in $H_{1}$. If instead we try to collapse first the vertices depicted with black circles in $H_{1}$, we obtain a graph that does not belong to $\mathrm{TW}_{2}$.

\section{Conservativity arguments}

We now show that the results presented in the previous sections extend to pure allegories (without top). We do so by proving that $A l l_{\top}$ is a conservative extension of $A l l$, i.e., that for all terms $u, v$ in the syntax of allegories (i.e., for all T-free terms), $\vdash_{A l l_{\top}} u=v$ if and only if $r_{\text {All }} u=v$. Consequently, the equational theory of allegories is decidable and Theorem 16 also holds for All.

We prove conservativity by showing that every allegory embeds into an allegory with top (Proposition 44). It happens that we can factorise this construction to show in passing how to handle isomorphisms of connected $\mathrm{K}_{4}$-free graphs. This is what we do first.

Proofs in this section are mostly equational and often involve many cases. We present sketches to give intuitions; more details can been found in Appendix D; full proofs formalised in Coq can be browsed online [21].

\subsection{Isomorphisms of connected graphs in $\mathrm{TW}_{2}$}

As explained in [7], connected $\mathrm{K}_{4}$-free graphs correspond to terms over the signature $\Sigma_{\text {dom }}$, where $T$ is no longer present, and dom() becomes a primitive operation. It was however left open whether isomorphisms of such graphs could be finitely axiomatised over this syntax. We answer this question by the affirmative.

Definition 37. A 2pdom-algebra is a $\Sigma_{\text {dom-algebra satisfying the }}$ axioms (A1-A8) from Figure 4 except (A3), and the axioms in Figure 8 . We write $\vdash_{2 p d o m} u=v$ when two terms $u$ and $v$ are congruent modulo those axioms.

$$
\begin{aligned}
1 \cap 1 & =1 \\
\operatorname{dom}(u \cap v) & =1 \cap u \cdot v^{\circ} \\
\operatorname{dom}(u \cdot v) & =\operatorname{dom}(u \cdot \operatorname{dom}(v)) \\
\operatorname{dom}(u) \cdot(v \cap w) & =\operatorname{dom}(u) \cdot v \cap w
\end{aligned}
$$

Figure 8. Axioms for 2pdom-algebras (with (A1,A2,A4-A8)).

Graphs form a 2pdom-algebra: the axioms are sound. To prove that they are complete for graph isomorphisms, we rely on Theorem 18 and we prove that every $2 p d o m$ algebra can be embedded in a $2 p$ algebra. Combined with the other results from [7], this also yields that connected $\mathrm{K}_{4}$-free graphs form the free $2 p d o m$-algebra.

We fix a $\Sigma_{\text {dom }}$-algebra $\left\langle X, \cdot, \cap,{ }_{-}^{\circ}, \operatorname{dom}\left(\_\right), 1\right\rangle$ in the remainder of this section. We write $T$ for the set of tests in $X$. We construct the following $\Sigma_{\top}$-algebra:

Definition 38. Let $\bar{X}$ be the set $X \uplus T^{2}$. For $u \in X$, we write $\bar{u}$ for $u$ as an element of $\bar{X}$. For $\alpha, \beta \in T$, we write $\alpha \diamond \beta$ for the pair $\langle\alpha, \beta\rangle$ as an element of $\bar{X}$. We turn $\bar{X}$ into a $\Sigma_{\mathrm{T}}$-algebra by setting:

$$
\begin{array}{cr}
\bar{u} \cdot \bar{v} \triangleq \overline{u \cdot v} & \bar{u} \cap \bar{v} \triangleq \overline{u \cap v} \\
(\alpha \diamond \beta) \cdot(\gamma \diamond \delta) \triangleq \alpha \diamond \delta & (\alpha \diamond \beta) \cap(\gamma \diamond \delta) \triangleq \alpha \gamma \diamond \beta \delta \\
(\alpha \diamond \beta) \cdot \bar{v} \triangleq \alpha \diamond \operatorname{dom}\left(v^{\circ} \beta\right) & (\alpha \diamond \beta) \cap \bar{v} \triangleq \overline{\alpha v \beta} \\
\bar{u} \cdot(\gamma \diamond \delta) \triangleq \operatorname{dom}(u \gamma) \diamond \delta & \bar{u} \cap(\gamma \diamond \delta) \triangleq \overline{\gamma u \delta} \\
(\alpha \diamond \beta)^{\circ} \triangleq \beta \diamond \alpha & 1 \triangleq \overline{1} \\
\bar{u}^{\circ} \triangleq \overline{u^{\circ}} & \top \triangleq 1 \diamond 1
\end{array}
$$

When $X$ is the algebra of connected graphs, $\bar{X}$ intuitively represent graphs where all vertices are connected either to the input or to the output: an element $\bar{u}$ denotes a connected graph, while an element $\alpha \diamond \beta$ denotes the disconnected graph $\alpha \top \beta$.

When composing two 'disconnected elements' $\alpha \diamond \beta$ and $\gamma \diamond \delta$ in series, we throw away a component that should intuitively be created and which is not connected to the input or to the output: $\beta \gamma$. This means that $\bar{X}$ cannot be the free $2 p$-algebra: whatever the starting $2 p d o m$-algebra $X, \bar{X}$ always satisfies the law $\mathrm{\top} u \top=\mathrm{\top}$.

Note however that the function mapping an element $u \in X$ to $\bar{u}$ is an injective $\Sigma$-homomorphism from $X$ to $\bar{X}$.

Lemma 39. In $2 p d o m$, an element $u$ is a test iff $\operatorname{dom}(u)=u$.

Proposition 40. If $X$ is a 2pdom-algebra then $\bar{X}$ is a 2p-algebra.

Proof. We must show that $\bar{X}$ satisfies all the $2 p$ axioms (Figure 6). Consider the associativity of product (A4). Each of the three variables occurring in this axiom can be either in $X$ or in $T^{2}$. The proof is trivial when all elements are in $X$. We show two relevant cases.

$$
\begin{aligned}
((\alpha \diamond \beta) \cdot \bar{u}) \cdot(\gamma \diamond \delta) & =\left(\alpha \diamond \operatorname{dom}\left(u^{\circ} \beta\right)\right) \cdot(\gamma \diamond \delta) \\
& =\alpha \diamond \delta \\
(\alpha \diamond \beta) \cdot(\bar{u} \cdot(\gamma \diamond \delta)) & =(\alpha \diamond \beta) \cdot(\operatorname{dom}(u \gamma) \diamond \delta) \\
((\alpha \diamond \beta) \cdot \bar{u}) \cdot \bar{v} & =\left(\alpha \diamond \operatorname{dom}\left(u^{\circ} \beta\right)\right) \cdot \bar{v} \\
& =\alpha \diamond \operatorname{dom}\left(v^{\circ} \operatorname{dom}\left(u^{\circ} \beta\right)\right) \\
& =\alpha \diamond \operatorname{dom}\left(v^{\circ} u^{\circ} \beta\right) \\
(\alpha \diamond \beta) \cdot(\bar{u} \cdot \bar{v}) & =(\alpha \diamond \beta) \cdot \overline{u v}
\end{aligned}
$$


Corollary 41. For all $\Sigma_{\mathrm{dom}}$-terms $u, v$ we have

$$
r_{2 p d o m} u=v \quad \text { iff } \quad r_{2 p} u=v \text { iff } \mathrm{g}(u) \simeq \mathrm{g}(v) \text {. }
$$

\subsection{Pure allegories}

We now prove that $A l l_{\top}$ is a conservative extension of $A l l$, using the same construction as in Definition 38.

A difficulty here is that we need a domain operation in order to use this construction, and this operation is not in the syntax of allegories, nor derivable due to the absence of $T$. Fortunately, we can use the alternative operation $\operatorname{dom}^{\prime}(\cdot)\left(\right.$ defined by $\operatorname{dom}^{\prime}(u) \triangleq$ $\left.1 \cap u u^{\circ}\right)$. Indeed, these terms are equivalent in allegories with $T$ (Equation (2) from Section 4).

The following lemma makes it possible to reuse Proposition 40 in the proof of Proposition 44 below.

Lemma 42. If $\left\langle X, \cdot, \cap,{ }_{-}^{\circ}, 1\right\rangle$ is an allegory, then $\left\langle X, \cdot, \cap,{ }_{-}^{\circ}, \operatorname{dom}^{\prime}(\cdot), 1\right\rangle$ is a 2pdom-algebra.

Proof. It suffices to show that the axioms in Figure 8 are derivable in All. (A9) is an instance of idempotency; (A10) and (A13) have been proved in Proposition 14; (A14) follows from law (4) in Proposition 14 since $\operatorname{dom}^{\prime}(u)$ is by definition a test in All.

Lemma 43. Let $X$ be an allegory; the partial order derived on $\bar{X}$ is characterised in terms of the partial order on $X$ as follows.

$$
\begin{array}{cl}
\bar{u} \geq \bar{v} & \text { iff } u \geq v \\
\alpha \diamond \beta \geq \gamma \diamond \delta & \text { iff } \alpha \geq \gamma \text { and } \beta \geq \delta \\
\alpha \diamond \beta \geq \bar{v} & \text { iff } \alpha v \beta \geq v \\
\bar{u} \geq \gamma \diamond \delta & \text { never holds }
\end{array}
$$

Proposition 44. If $X$ is an allegory then $\bar{X}$ is an allegory with top.

Proof. By Lemma 42 and Proposition 40, it suffices to prove idempotency, semi-distributivity, and modularity. We show two interesting cases for modularity.

- $\left((\alpha \diamond \beta) \cap \overline{w v}^{\circ}\right) \bar{v} \geq(\alpha \diamond \beta) \bar{v} \cap \bar{w}$ : we have

$$
\begin{aligned}
\left((\alpha \diamond \beta) \cap \overline{w v}^{\circ}\right) \bar{v}= & \overline{\alpha w v^{\circ} \beta v} \\
(\alpha \diamond \beta) \bar{v} \cap \bar{w}= & \overline{\alpha w \operatorname{dom}^{\prime}\left(v^{\circ} \beta\right)}, \text { and } \\
\alpha w v^{\circ} \beta v \geq \alpha w\left(1 \cap v^{\circ} \beta v\right) & =\alpha w\left(1 \cap v^{\circ} \beta^{\circ} \beta v\right) \\
& =\alpha w\left(\operatorname{dom}^{\prime}\left(v^{\circ} \beta\right)\right)
\end{aligned}
$$

- $\left(\bar{u} \cap \bar{w}(\alpha \diamond \beta)^{\circ}\right)(\alpha \diamond \beta) \geq \bar{u}(\alpha \diamond \beta) \cap \bar{w}$ : we have

$$
\begin{aligned}
\left(\bar{u} \cap \bar{w}(\alpha \diamond \beta)^{\circ}\right)(\alpha \diamond \beta) & =\operatorname{dom}^{\prime}\left(\operatorname{dom}^{\prime}\left(w \beta^{\circ}\right) u \alpha^{\circ} \alpha\right) \diamond \beta \\
\bar{u}(\alpha \diamond \beta) \cap \bar{w} & =\overline{\operatorname{dom}^{\prime}(u \alpha) w \beta}
\end{aligned}
$$

We use Lemma 43 and prove

$$
\begin{aligned}
& \operatorname{dom}^{\prime}\left(\operatorname{dom}^{\prime}\left(w \beta^{\circ}\right) u \alpha^{\circ} \alpha\right) \operatorname{dom}^{\prime}(u \alpha) w \beta \beta \\
= & \operatorname{dom}^{\prime}(u \alpha) \operatorname{dom}^{\prime}(w \beta) w \beta \\
\geq & \operatorname{dom}^{\prime}(u \alpha) w \beta
\end{aligned}
$$

where the last step follows by $\vdash_{A l l} \operatorname{dom}^{\prime}(u) u \geq u$ :

$$
\operatorname{dom}^{\prime}(u) u=\left(1 \cap u u^{\circ}\right) u \geq 1 u \cap u=u \quad \text { (by (AM)) }
$$

Corollary 45. For all $\Sigma$-terms $u, v$ we have $\vdash_{\text {All }} u=v$ if and only if $\vdash_{A l l_{\top}} u=v$.

Decidability of allegories follows, as well as the expected graphtheoretical characterisation:

Corollary 46. We have $r_{A l l} u=v$ if and only if $\mathrm{g}(u) \rightleftharpoons \mathrm{g}(v)$.

\section{Future work}

We proved decidability (in NP) of the equational theories of allegories and allegories with top, and we designed a graph rewriting system making it possible to compute normal forms. The precise complexity of these equational theories remains open.

There is a simple polynomial algorithm to find whether there is a (arbitrary) homomorphism from $\mathrm{g}(u)$ to $\mathrm{g}(v)$ : fix $G=\mathrm{g}(v)$ and consider the following formula over subterms $w$ of $u$ and pairs $j, k$ of vertices in $G$ :

$$
\phi(w, j, k) \triangleq \text { there is a homomorphism from } \mathrm{g}(w) \text { to } G[j, k]
$$

Consider the truth table of this formula, i.e., the table indexed by subterms of $u$ and pairs of vertices in $G$, whose cells contain true or false depending on the validity of the formula $\phi$. This table can be computed recursively for all arguments, in polynomial time (dynamic programming). However, it is not clear whether this algorithm could be adapted to compute the restricted relation efficiently.

\section{Acknowledgments}

We would like to thank the anonymous reviewers for their comments and Michael Winter for pointing us to useful references.

\section{References}

[1] B. Aameri and M. Winter. A first-order calculus for allegories. In Proc. RAMiCS, pages 74-91, Berlin, Heidelberg, 2011. Springer.

[2] H. Andréka and D. A. Bredikhin. The equational theory of union-free algebras of relations. Algebra Universalis, 33(4):516-532, 1995.

[3] H. Andréka and S. Mikulás. Axiomatizability of positive algebras of binary relations. Algebra Universalis, 66(1):7-34, 2011.

[4] C. Brown and G. Hutton. Categories, allegories, and circuit design. In Proc. LICS. IEEE, 1994.

[5] A. K. Chandra and P. M. Merlin. Optimal implementation of conjunctive queries in relational data bases. In Proc. STOC, pages 77-90. ACM, 1977.

[6] C. Chekuri and A. Rajaraman. Conjunctive query containment revisited. Theoretical Computer Science, 239(2):211-229, 2000

[7] E. Cosme-Llópez and D. Pous. K4-free graphs as a free algebra. In Proc. MFCS, volume 83 of LIPIcs, pages 76:1-76:14. Schloss Dagstuhl, 2017.

[8] B. Davey and H. Priestley. Introduction to Lattices and Order. Cambridge University Press, 2nd (2002) edition, 1990.

[9] R. Diestel. Graph Theory. Graduate Texts in Mathematics. Springer, 2005.

[10] R. Duffin. Topology of series-parallel networks. Journal of Mathematical Analysis and Applications, 10(2):303-318, 1965.

[11] E. C. Freuder. Complexity of k-tree structured constraint satisfaction problems. In Proc. NCAI, pages 4-9. AAAI Press / The MIT Press, 1990

[12] P. Freyd and A. Scedrov. Categories, Allegories. North Holland. Elsevier, 1990.

[13] J. Glanfield. Towards automated derivation in the theory of allegories. MSc. Thesis, Brock University, 2008

[14] M. Grohe. The complexity of homomorphism and constraint satisfaction problems seen from the other side. Fournal of the ACM, 54(1):1:1-1:24, Mar. 2007.

[15] C. Gutierrez. Decidability of the equational theory of allegories. In Proc. RelMiCS, 1998.

[16] C. Gutierrez. The Arithmetic and Geometry of Allegories: Normal Forms and Complexity of a Fragment of the Theory of Relations. PhD thesis, Wesleyan University, Middletown, CT, USA, 1999. AAI9934518.

[17] P. Hell and J. Nešetril. The core of a graph. Discrete Mathematics, 109(1):117 126, 1992.

[18] P. T. Johnstone. Sketches of an elephant: a Topos theory compendium. Oxford logic guides. Oxford Univ. Press, New York, NY, 2002

[19] D. Kozen. Kleene algebra with tests. Transactions on Programming Languages and Systems, 19(3):427-443, May 1997.

[20] D. Pous and V. Vignudelli. Allegories: decidability and graph homomorphisms. In Proc. LiCS. ACM, 2018.

[21] D. Pous and V. Vignudelli. Web appendix to this paper, including Coq proofs of all equational proofs and conservativity results, 2018 . http://perso.ens-lyon.fr/damien.pous/allegories/.

[22] N. Robertson and P. Seymour. Graph minors. XX. Wagner's conjecture. fournal of Combinatorial Theory, Series B, 92(2):325 - 357, 2004. 


\section{A Example in Figure 1}

The homomorphism from the first to the second graph in Figure 1 corresponds to an inequality provable in All, i.e., it is of the form $\rightarrow$. We show that indeed the homomorphism can be decomposed as a sequence of homomorphisms on graphs in $\mathrm{TW}_{2}$ merging two vertices at a time. Among the vertices having the same shape and colour in the first graph

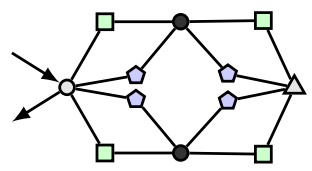

the only two vertices that we can collapse are the ones depicted with a black circle. Otherwise, we would obtain a graph whose strong skeleton has $\mathrm{K}_{4}$ as a minor. If we do so, we obtain

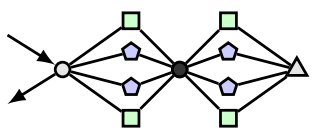

Then we can merge in sequence the following pairs of vertices: the two green squares on the left, the two blue pentagons on the left, the two green squares on the right, the two blue pentagons on the right. At each step, the reached graph is a subgraph of the previous one, and thus is in $\mathrm{TW}_{2}$ (it actually does not matter the order in which we merge these pairs). After applying these four homomorphisms, we have

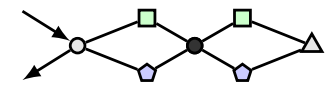

We can now collapse the two remaining green squares and obtain a graph in $\mathrm{TW}_{2}$ (symmetrically, we could have first collapsed the blue pentagons)

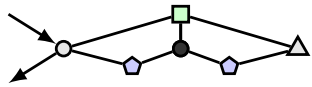

Finally, we collapse the green squares and obtain the target graph

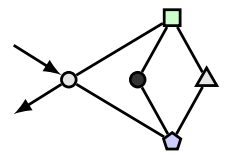

This concludes the proof that $G_{1} \rightarrow G_{2}$.

By contrast, the third graph in Figure 1

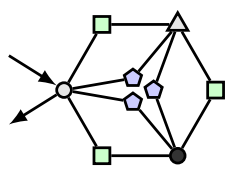

has a homomorphism to $G_{2}$, but the graphs are not related by $\rightarrow$. Since $\rightarrow=\succ^{*} \hookrightarrow$ (Proposition 32), in order to prove $G_{3} \nrightarrow G_{2}$ it suffices to note that whenever we collapse two nodes with the same shape in $G_{3}$ we obtain a graph not in $\mathrm{TW}_{2}$; the following one for instance.

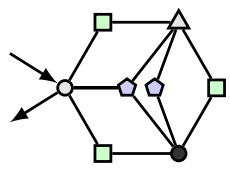

We conclude that $G_{3} \not G_{2}$ : there is no injection from $G_{3}$ to $G_{2}$.

\section{B Leaving/entering $\mathrm{TW}_{2}$ by merging vertices}

Let $G$ be the graph depicted below, and let $G^{\prime}$ be the graph obtained by collapsing the nodes connected by the dotted line.

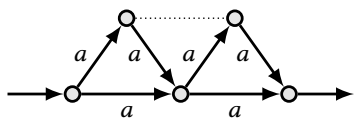

The function $g: G \rightarrow G^{\prime}$ that assigns the vertices connected by the dotted line to the merged node and that is injective on the other nodes is a surjective homomorphism equating only two vertices. Graph $G$ in $\mathrm{TW}_{2}$, but $G^{\prime}$ is not.

Let $H$ be the following graph

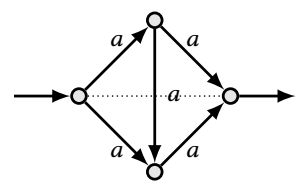

and let $H^{\prime}$ be the graph obtained by collapsing the nodes connected by the dotted line. As in the previous example, there is a surjective homomorphism $h: H \rightarrow H^{\prime}$ equating only two vertices. In this case, graph $H$ is not in $\mathrm{TW}_{2}$, but $H^{\prime}$ is.

\section{Confluence of the relation $\leadsto$}

We prove confluence of relation $\leadsto$ (Proposition 36). The proof relies on the following lemma.

Lemma 47. Let $G_{1}, G_{2}$ be graphs in $\mathrm{TW}_{2}$. If $G_{1} \rightleftharpoons G_{2}$ then there is an $H$ such that $G_{1} \sim H$ and $G_{2} \sim H$.

Proof. We prove the statement by induction on $s z\left(G_{1}\right)+s z\left(G_{2}\right)$, where $s z(G)$ is the sum of the number of nodes and edges in $G$. By the definition of $G_{1} \rightleftharpoons G_{2}$ we have that there exist $G_{1}^{\prime}, G_{2}^{\prime}$ such that

$$
G_{1} \succ^{*} G_{1}^{\prime} \hookrightarrow G_{2} \succ^{*} G_{2}^{\prime} \hookrightarrow G_{1}
$$

By $G_{1}^{\prime} \hookrightarrow G_{2} \succ^{*} G_{2}^{\prime}$ we derive, by Proposition 32 , that there is a $G_{3}$ such that $G_{1}^{\prime} \succ^{*} G_{3} \hookrightarrow G_{2}^{\prime}$. Hence, $G_{1} \leadsto G_{3}$, which also implies $s z\left(G_{1}\right) \geq s z\left(G_{3}\right)$. If $s z\left(G_{1}\right)>s z\left(G_{3}\right)$, by $G_{3} \rightleftharpoons G_{2}$ we can apply the inductive hypothesis and derive that there is an $H$ such that $G_{3} \leadsto H$ and $G_{2} \leadsto H$. Since $\leadsto$ is transitive, we have $G_{1} \leadsto H$.

Otherwise, suppose $s z\left(G_{1}\right)=s z\left(G_{3}\right)$. Then $G_{1} \simeq G_{1}^{\prime} \simeq G_{3}$, which implies $G_{1} \hookrightarrow G_{2}$. Hence, $G_{2} \leadsto G_{2}^{\prime}$ and either $s z\left(G_{2}\right)>s z\left(G_{2}^{\prime}\right)$, in which case we conclude by the inductive hypothesis and by the transitivity of $\leadsto$, or $s z\left(G_{2}\right)=s z\left(G_{2}^{\prime}\right)$, in which case $G_{1} \simeq G_{2}$.

We deduce confluence of $\leadsto$ as follows: if $G \leadsto G_{1}$ and $G \leadsto G_{2}$ then $G_{1} \rightleftharpoons G_{2}$, and by Lemma 47 we have that there is an $H$ such that $G_{1} \leadsto H$ and $G_{2} \leadsto H$.

\section{Additional proofs for Section 8}

Proof of Lemma 39. If $u=1 \cap u$ then we have

$$
\operatorname{dom}(u)=\operatorname{dom}(u \cap 1)=1 \cap u 1^{\circ}=1 \cap u=u
$$

Conversely, we have

$$
\begin{aligned}
\operatorname{dom}(u) & =\operatorname{dom}(u)(1 \cap 1) \\
& =\operatorname{dom}(u) 1 \cap 1 \\
& =1 \cap \operatorname{dom}(u)
\end{aligned}
$$


Proof of Lemma 43. The first item follows from the definition. We have

$$
\begin{array}{rll}
\alpha \diamond \beta \geq \gamma \diamond \delta \quad \text { iff } & \alpha \diamond \beta \cap \gamma \diamond \delta=\gamma \diamond \delta \\
& \text { iff } \quad \alpha \gamma \diamond \beta \delta=\gamma \diamond \delta \\
& \text { iff } \quad \alpha \gamma=\gamma \text { and } \beta \delta=\delta \\
& \text { iff } \quad \alpha \cap \gamma=\gamma \text { and } \beta \cap \delta=\delta \quad \text { (by (5)) } \\
\text { iff } & \alpha \geq \gamma \text { and } \beta \geq \delta \\
\alpha \diamond \beta \geq \bar{v} & \text { iff } & \alpha \diamond \beta \cap \bar{v}=\bar{v} \\
& \text { iff } \quad \alpha v \beta \cap \bar{v}=\bar{v} \\
& \text { iff } \quad \alpha v \beta \cap v=v \\
& \text { iff } & \alpha v \beta \geq v
\end{array}
$$

Finally, $\bar{u} \geq \gamma \diamond \delta$ holds iff $\overline{\gamma u \delta}=\gamma \diamond \delta$, which never holds.

\section{E Problems with the approach in [15]}

We list the difficulties we encountered when trying to follow the strategy proposed in [15].

1. [15, Theorem 1] claims that a certain axiomatisation over the signature $\Sigma$ is sound and complete for graph isomorphisms. No proof is given and we do not know how to obtain this result. We needed a full paper [7] to prove a similar claim for the signature $\Sigma_{\mathrm{T}}$ (Theorem 18), and the conservativity technique we used in Section 8.1 to get one for the signature $\Sigma_{\text {dom. }}$. It is not clear whether this technique could scale to prove [15, Theorem 1] using the result in [7]: our construction requires a domain operator.

2. It is not clear how the conditions of [15, Definition 6] should be applied to graphs that are tests.

3. [15, Theorem 2] (soundness and completeness of a graphtheoretical notion w.r.t allegories) comes with a five-line proof sketch. We do not know how to complete this sketch for the following reasons:

- For the 'only if' direction, it is not clear that the notion of walk in a graph is closed under contexts (i.e., whether a counter-part to Lemma 27 holds).

- The 'if' part relies on [15, Proposition 1], which is announced to hold by induction. We do not see how to deal with the case of composition in this induction: the induction hypothesis does not seem to be strong enough.

4. [15, Lemma 2] is used to define a notion of normal forms, which should then lead to decidability. It is however not clear that those normal forms are computable. While the set mentioned in this Lemma is indeed finite (it consists of subgraphs of a given graph), one has to consider infinitely many sequences (the arrows of the category $\mathrm{G}(X)$ ) in order to decide membership in this set. 